\title{
Diretivas antecipadas de vontade em pacientes com doença de Alzheimer
}

Luciana Dadalto $^{1}$, Alexandra Mendes Barreto Arantes $^{2}$, Priscila Demari Baruffi ${ }^{3}$

1. Centro Universitário Newton Paiva, Belo Horizonte/MG, Brasil. 2. Escola Superior em Ciências da Saúde, Brasília/DF, Brasil.

3. Universidade de Caxias do Sul, Caxias do Sul/RS, Brasil.

\section{Resumo}

Em todo o mundo a população idosa tem crescido e, juntamente com o envelhecimento populacional, nota-se o aumento da incidência de demências. Sob a ótica da importância dos instrumentos de manifestação de vontade, e mais especificamente das diretivas para demências, este artigo analisa fatores que contribuem com a perda de autonomia dos idosos acometidos pela doença de Alzheimer em sua fase mais avançada. Para que possa esclarecer seus desejos, é necessário que o idoso estabeleça diretivas preferencialmente na fase inicial da doença, ou seja, enquanto tem capacidade de decidir por si, manifestando suas opiniões e preferências. Conclui-se, no entanto, que ainda é preciso mais debates para que esse instrumento seja usado da melhor forma possível, garantindo a autodeterminação dos pacientes.

Palavras-chave: Envelhecimento. Autonomia pessoal. Doença de Alzheimer. Diretivas antecipadas.

\section{Resumen}

Directivas anticipadas de voluntad en pacientes con enfermedad de Alzheimer

En todo el mundo, la población de adultos mayores ha crecido y, junto con el envejecimiento de la población, la incidencia de la demencia ha aumentado. Desde la perspectiva de la importancia de los instrumentos de manifestación de Voluntad, y más específicamente de las directivas para las demencias, este artículo analiza factores que contribuyen a la pérdida de autonomía de los adultos mayores afectados por la enfermedad de Alzheimer en su etapa más avanzada. Para que él pueda aclarar sus deseos, es necesario que el adulto mayor establezca directrices preferiblemente en la etapa inicial de la enfermedad, es decir, mientras tenga la capacidad de decidir por sí mismo, expresando sus opiniones y preferencias. Se concluye, sin embargo, que aún se necesitan más debates para que este instrumento sea utilizado de la mejor manera posible, asegurando la autodeterminación de los pacientes.

Palabras-clave: Envejecimiento. Autonomía personal. Enfermedad de Alzheimer. Directivas anticipadas.

\section{Abstract}

\section{Advance directives for patients with Alzheimer's disease}

The older population is experiencing worldwide growth, and together with population aging the incidence of dementia is also increasing. This article examines the contributing factors to the loss of autonomy of older people affected by Alzheimer's disease in its most advanced stage based on the importance of instruments for expression of will, especially the advance directive for dementia. In order for older people with Alzheimer's disease to clarify their wishes, they need to prepare their directives preferably in the early stages of the disease, that is, when they still have the capacity to make autonomous decisions, expressing their values and preferences. However, further discussions on this issue are necessary for these instruments to be better used in the Brazilian context, and thus ensure the patients' self-determination.

Keywords: Aging. Personal autonomy. Alzheimer disease. Advance directives. 
Independentemente de sua idade, uma pessoa pode ser considerada saudável quando é capaz de efetuar suas atividades sozinha, sem dependência, mesmo que apresente alguma doença ${ }^{1}$. Entre os idosos, "estar saudável" depende mais da condição de autonomia e independência do que da presença ou não de patologias. No Brasil, pessoas com idade igual ou superior a 60 anos são consideradas idosas com base na Lei $10.741 / 2003^{2}$, conhecida como Estatuto do Idoso, que regula direitos assegurados e dá prioridade de atendimento a essa população, tratando o envelhecimento como direito personalíssimo.

As doenças crônicas, que frequentemente acometem idosos, contribuem para maior incapacidade e mortalidade ${ }^{3}$. Associadas muitas vezes a essas doenças, as demências decorrem de fatores e enfermidades pré-existentes e geralmente têm curso lento, afetando o sujeito acometido de várias formas: moral, comportamental ou intelectual $^{4}$. A doença de Alzheimer é a principal causa de demência e um dos maiores desafios em saúde do século XXI ${ }^{5}$. Devido a sua evolução, o enfermo pode se sentir incapaz de tomar decisões, o que o torna vulnerável $^{6}$. Trata-se de doença irreversível e progressiva, e os últimos anos do paciente são marcados por perda grave e persistente de funcionalidade.

Apesar de a demência ser extremamente frequente na velhice, atingindo 50 milhões de pessoas no mundo atualmente e com estimativa de se expandir para 150 milhões em $2050^{7}$, pouco se tem discutido sobre seu curso irreversível e a condição terminal. A consequência é que as discussões sobre o final da vida ainda são insuficientes, e idosos com demência em fase avançada têm recebido tratamentos desproporcionais (levando em conta a ausência de benefícios e de mudança do curso da doença, além de riscos de danos) ${ }^{8}$.

Considerando a evolução inexorável da doença de Alzheimer, a necessidade de cuidados paliativos se torna necessária desde o diagnóstico. Segundo a Organização Mundial da Saúde $(\mathrm{OMS})^{9}$, tais cuidados são uma abordagem que visa controlar sintomas e garantir mais conforto e qualidade de vida. Ainda conforme a OMS, a assistência paliativa deve ser oferecida em conjunto com o tratamento-padrão de qualquer doença que ameace a continuidade da vida?.

De acordo com a International Association for Hospice and Palliative Care ${ }^{10}$, cuidados paliativos são cuidados holísticos ativos, ofertados a pessoas de todas as idades, especialmente em fim de vida, que se encontram em intenso sofrimento proveniente de doença grave. $O$ objetivo desses cuidados é melhorar a qualidade de vida dos pacientes, de suas famílias e de seus cuidadores.

Em 2014, a Sociedade Europeia de Cuidados Paliativos fez recomendações de melhores práticas de cuidados paliativos para idosos com demên$\mathrm{cia}^{11}$. Dentre as recomendações, destacam-se: os problemas apresentados pelo paciente devem ser vistos pela perspectiva do paciente; na demência leve, os pacientes precisam de auxílio para planejar o futuro; nas fases mais avançadas, o melhor interesse do paciente deve ser o objetivo do cuidado; e o plano avançado deve ser um processo regularmente revisado. As diretrizes vão ao encontro do cuidado centrado no paciente e reconhecem a importância da autonomia.

Dadalto $^{12}$ explica que, motivado pelas particularidades apresentadas pelas demências, o médico geriatra Barak Gaster publicou estudo sobre manifestações prévias de cuidados de saúde, defendendo que as demências mereciam ter um documento específico, por ele chamado de "diretivas antecipadas para demências" (DAD), que as retirasse de outras diretivas. No testamento vital, por exemplo, o paciente se manifesta de maneira autônoma, porém, na opinião de Gaster ${ }^{12}$, no caso de pacientes com demência deveria haver interferência e assistência de terceiros que podem ser impactados por aquela doença no futuro.

O presente artigo busca contextualizar as DAD como instrumento capaz de resguardar a autonomia do idoso com demência.

\section{Envelhecimento ou Alzheimer?}

Paira na sociedade brasileira a ideia equivocada de que a velhice traz somente dificuldades à vida do indivíduo, tornando-a recheada de perdas e total dependência. Todavia, esse pensamento só existe por conta da interpretação errônea da velhice e da doença como termos idênticos ${ }^{13}$. $O$ envelhecimento cerebral normal pode causar lapsos rotineiros (por exemplo, não se lembrar se a porta do carro foi trancada), mas tais lapsos, quando não comprometem a funcionalidade, podem ser considerados falta de atenção, e não de memória ${ }^{14}$. 
Com o natural processo de envelhecimento, o cérebro humano acaba perdendo uma parcela da massa encefálica, mas as funções mentais permanecem preservadas até a morte do indivíduo. Logo, é possível que o idoso chegue a essa fase da vida com saúde física e mental. Para isso, a fim de manter a autonomia, é preciso se manter ativo ${ }^{4}$. Por outro lado, quando acometido por demências, o indivíduo perde progressivamente suas funções cognitivas, o que prejudica sua capacidade de independência e autonomia.

A doença de Alzheimer e outras demências são subdiagnosticadas e, mesmo quando o diagnóstico ocorre, ele é frequentemente omitido ${ }^{15}$. Cerca de $50 \%$ dos portadores da doença não descobrem a patologia no estágio inicial ou, quando a descobrem, não a tratam, desprezando inclusive as consultas médicas, que poderiam influenciar o curso da doença, já que ela é progressiva e tem diversas fases, da mais leve até a mais avançada ${ }^{16}$. No entanto, o atraso no diagnóstico muitas vezes faz com que o paciente chegue a um profissional já sem capacidade de tomar decisões.

Muitas são as descobertas recentes a respeito da memória e do cérebro humano, e as pesquisas nessa área têm motivado diversos avanços e tentativas de tratamento. A cura para o Alzheimer, porém, ainda não foi encontrada, e o diagnóstico definitivo só pode ser feito por meio de biópsia ou necropsia ${ }^{17}$.

A doença acomete indivíduos idosos, na maior parte mulheres com 65 anos ou mais ${ }^{16}$. No entanto, há também a doença de Alzheimer de início precoce, que ocorre em indivíduos de faixas etárias anteriores. Na família das demências, a doença de Alzheimer corresponde à elevada porcentagem de $60 \%$ ou $70 \%$ dos diagnósticos ${ }^{16}$.

De forma lenta, e muitas vezes sem que os próprios familiares percebam, a doença de Alzheimer modifica o comportamento do indivíduo. A apatia, por exemplo, é um sintoma comum no quadro clínico ${ }^{16}$. Além da memória, a enfermidade compromete habilidades manuais (por exemplo, pintura) e funções executivas, como planejar e fazer compras no supermercado.

A linguagem do acometido também pode ficar confusa, dificultando a comunicação com familiares e cuidadores ${ }^{17}$. Na fase avançada da doença, o idoso pode até mesmo não conseguir expressar suas preferências e valores para que a equipe de saúde alinhe suas condutas e honre as vontades do paciente.

\section{Autonomia da vontade no fim da vida}

A saúde e a integridade física dos indivíduos devem ser pautadas por princípios e valores como autonomia, beneficência e não maleficência. A autonomia - que compreende o ser como agente dotado de racionalidade e liberdade, capaz de fazer suas próprias escolhas ${ }^{18}$ - tem papel importante até o fim da vida do paciente. Ela assegura os direitos fundamentais do indivíduo e sua dignidade, visto que, na perspectiva jurídica, o princípio constitucional da dignidade da pessoa humana é corolário do princípio da autonomia privada ${ }^{4}$. No que se refere especificamente aos idosos, a Lei $8.842 / 1994{ }^{19}$ criou o Conselho Nacional do Idoso a fim de assegurar os direitos sociais dessa população, sobretudo sua autonomia.

Conforme o caput do artigo $1^{\circ}$ da Resolução do Conselho Federal de Medicina (CFM) 1.805/2006 ${ }^{20}$, o médico pode limitar ou suspender procedimentos e tratamentos que prolonguem a vida do doente em fase terminal, com enfermidade grave e incurável, respeitada a vontade da pessoa ou de seu representante legal. Dessa forma, o médico deve sempre respeitar a autonomia do paciente, mesmo que em fase terminal. Caso o enfermo não possa expressar sua vontade, seu representante legal assumirá essa prerrogativa.

É imprescindível que o médico, antes de tomar suas decisões, informe o paciente sobre os procedimentos que podem ser adotados. O profissional deve prestar informações referentes às condições clínicas do paciente e às alternativas terapêuticas, considerando as chances de sucesso, riscos e possibilidade de sequelas ou complicações. A comunicação deve garantir a compreensão do paciente ou de seu representante legal, visto que esse entendimento é um dos pressupostos do consentimento necessário para realizar procedimentos ou tratamentos ${ }^{21}$.

As discussões acerca da ética médica têm deixado de se restringir aos profissionais da saúde, tornando-se objeto de discussões multidisciplinares $^{18}$. Dentre outras funções, a equipe multidisciplinar cumpre o papel de ajudar na transmissão do diagnóstico, facilitar a aceitação do tratamento, aliviar efeitos secundários e incentivar mudanças no estilo de vida dos pacientes ${ }^{22}$.

No estágio avançado da doença de Alzheimer e de outras patologias similares, os pacientes encontram-se impossibilitados de manifestar sua 
vontade, mas nem por isso tornam-se menos ativos em suas decisões ${ }^{4}$. O Código Civil brasileiro, em seu artigo $3^{\circ}$, define como absolutamente incapaz de exercer pessoalmente os atos da vida civil os indivíduos menores de 16 anos, e em seu artigo $4^{\circ}$ classifica os sujeitos incapazes relativamente a certos atos: os maiores de 16 anos, mas menores de 18 anos; os ébrios habituais e os viciados em tóxico; aqueles que, por causa transitória ou permanente, não puderem exprimir sua vontade; e os pródigos ${ }^{23}$.

O Estatuto da Pessoa com Deficiência, estabelecido pela Lei $13.146 / 2015^{24}$, assegura e promove condições de igualdade e exercício dos direitos e liberdades das pessoas com deficiência. Seu propósito é a inclusão social desses indivíduos, definidos como aqueles com impedimentos de longo prazo, de natureza física, mental, intelectual ou sensorial, que podem prejudicar a participação do sujeito na sociedade em comparação com indivíduos que não apresentam tais limitações ${ }^{24}$.

Assim, o Estatuto da Pessoa com Deficiência considera os indivíduos e suas capacidades de acordo com a diminuição da autonomia ${ }^{25}$. Segundo Sá e Oliveira ${ }^{26}$, presume-se que alguém é capaz até que o Estado, por meio do Poder Judiciário, o considere incapaz, limitando seus direitos civis. Outras formas de tutela do Estado são estabelecidas em normas específicas, como o Estatuto da Criança e do Adolescente, o Estatuto do Idoso e o próprio Estatuto da Pessoa com Deficiência.

Pessoas com capacidade cognitiva restrita para fornecer consentimento livre e esclarecido, como pessoas com deficiências mentais, devem ter um representante que apresente o consentimento ${ }^{25}$. Embora geralmente a capacidade de pacientes com demência esteja preservada no momento do diagnóstico, a maioria evoluirá para a perda dessa capacidade ${ }^{27}$. O princípio da autonomia ainda assim deverá ser protegido, pois garante não só a liberdade de pensamento e de ação de um indivíduo, mas faz com que outras pessoas participem e ajudem em seu desenvolvimento ${ }^{28}$.

Ainda que não se conheça com clareza o que experienciam os indivíduos acometidos por demência, investigações demonstram que, mesmo nas fases mais avançadas da doença, as pessoas continuam capazes de sentir emoções e de as transmitir por meio de comunicação não verbal ${ }^{29}$. Considerando a evolução da doença de Alzheimer, é necessário discutir a importância dos documentos de diretivas antecipadas de vontade como garantia de autonomia para esses pacientes.

\section{Diretivas antecipadas para demências}

Por envolver a biografia, a vontade, a vulnerabilidade e a autonomia da pessoa idosa, a doença de Alzheimer é complexa e provoca questionamentos que a bioética pode ajudar a responder ${ }^{30}$. É necessário discutir, por exemplo, a alteração de paradigmas médico-legais a fim de garantir a dignidade e a qualidade de vida do paciente, respeitando sua vontade mesmo quando ele não a pode expressar ${ }^{4}$.

No Brasil, a Resolução CFM 1.995/2012 aborda as diretivas antecipadas de vontade (DAV), definindo-as como o conjunto de desejos, prévia e expressamente manifestados pelo paciente, sobre cuidados e tratamentos que quer, ou não, receber no momento em que estiver incapacitado de expressar, livre e autonomamente, sua vontade ${ }^{31}$. A resolução tem como fundamento a discussão sobre a proporcionalidade de medidas no fim da vida.

Apesar do aumento do número de pacientes com doença de Alzheimer, são poucas as opções terapêuticas com evidência científica e houve intervalo de uma década para a descoberta de novas drogas. Todavia, a maioria desses medicamentos age apenas mitigando as alterações cognitivas e comportamentais, sem modificar a fisiopatologia da doença, que por isso é considerada incurável até o momento.

O curso prolongado do Alzheimer pode levar familiares e profissionais de saúde a não compreender o caráter incurável da doença ${ }^{32}$. Esse fato contribui para que a importância das DAV seja subestimada. No entanto, se aplicadas de maneira precoce, tais diretivas podem ser um instrumento para garantir a autonomia do paciente quando este já não for capaz de expressar seus valores e preferências.

As DAV só têm validade caso a pessoa esteja lúcida e consciente no momento de formulação do documento. Somente dessa forma as decisões registradas poderão ser consideradas quando o paciente não puder falar por si próprio ${ }^{30}$. O documento, desde que autorizado pelo paciente, deve ser anexado ao prontuário médico ou transcrito pelo médico. O profissional é objeto de fé pública, e seus atos têm efeito legal e jurídico, não sendo necessária a presença de testemunhas, tampouco assinatura ${ }^{33}$.

Há diferentes tipos de DAV: testamento vital (living will), procuração para cuidados de saúde (durable power attorney for health care), diretivas antecipadas psiquiátricas, diretivas 
antecipadas para demências, plano de parto e ordem de não reanimação ${ }^{12}$.

O testamento vital é um documento em que o sujeito capaz declara quais tratamentos médicos aceita ou rejeita caso futuramente se encontre impossibilitado de declarar sua vontade. Esse documento não deve conter nenhuma cláusula que vá contra o ordenamento jurídico ${ }^{34}$. Já a procuração para cuidados de saúde, conhecida também como "mandato duradouro", refere-se à nomeação de um procurador com poderes para decidir sobre tratamentos com base em instruções proferidas pelo paciente ${ }^{34}$.

As diretivas antecipadas psiquiátricas registram as preferências do paciente em tratamento psiquiátrico, para que essas diretrizes sejam aplicadas quando o sujeito eventualmente se encontrar com a capacidade prejudicada. O plano de parto, como o próprio nome sugere, trata de decisões da gestante diante de possíveis ocorrências no momento do parto. Já as ordens de não reanimação despontaram nos Estados Unidos nos anos 1970, quando começaram a ser divulgadas no protocolo de comunicação dos hospitais ${ }^{12}$.

As síndromes demenciais desafiam as DAV clássicas, dispostas no Patient Self Determination Act, lei americana da década de 1960. É necessário, portanto, um plano avançado de cuidados, que inclua as vontades da pessoa relativas a sua saúde a longo prazo, uma vez que essas síndromes tendem a ser duradouras ${ }^{30}$.

$\mathrm{Na}$ opinião de Assumpção ${ }^{35}$, o testamento vital é o instrumento que melhor se adequa à expressão dos anseios da pessoa, registrando de maneira mais segura as vontades de cada indivíduo e afastando eventuais dilemas familiares. Para Torres da Silva ${ }^{4}$, o testamento vital e o mandato duradouro são ferramentas jurídicas úteis para preservar a autonomia do indivíduo no que se refere a decisões médicas e situações de fim de vida. No entanto, o próprio autor destaca que, embora esses instrumentos possam suprir satisfatoriamente as necessidades de um paciente com Alzheimer ou outras demências, é preciso um documento detaIhado, tendo em vista a complexibilidade dessas patologias, que envolvem cuidadores e familiares, tornando-se também um problema social.

Para Gaster, Larson e Curtis ${ }^{36}$, diretrizes antecipadas padronizadas - dentre elas o testamento vital não são a melhor alternativa para pacientes com alguma demência, como o Alzheimer. Isso porque a doença tem particularidades que exigem atenção especial. Assim, os autores propõem um documento específico: as diretivas antecipadas para demências.

Gaster, Larson e Curtis ${ }^{36}$ destacam que as DAD devem ser ajustadas ao longo do desenvolvimento da doença. Isto é, deve-se ponderar a necessidade da prestação e da continuidade de tratamentos, considerando a evolução da patologia ${ }^{36}$. A fim de divulgar essa ferramenta de proteção à autonomia, os autores disponibilizam um modelo de acesso livre. No Brasil, porém, o instrumento ainda não foi validado.

Pacientes com Alzheimer têm níveis de perda de autonomia mais elevados que os demais idosos. A doença tem diversas fases, que conforme avançam aumentam a dependência do idoso em relação a outras pessoas, impedindo até mesmo tarefas rotineiras ${ }^{4}$. A Sociedade de Alzheimer Americana ${ }^{37}$ considera que, na fase inicial da doença, o indivíduo ainda entende o significado e a importância de documentos legais, tendo condições e capacidade legal para compreender as consequências de suas ações e registrar suas vontades.

Enquanto tiver capacidade legal, o paciente deve participar do planejamento de seu cuidado. Dessa forma, as DAD podem ser elaboradas antes, durante ou imediatamente após o diagnóstico de demência, desde que o paciente tenha a capacidade necessária. É importante enfatizar que o momento preciso de elaboração do documento é muito importante para sua validação ética ${ }^{38}$.

O Mental Capacity Act (MCA) $2005^{39}$ utilizado na Inglaterra e no País de Gales, fornece um protocolo para empoderar e proteger as pessoas que não têm capacidade de tomar decisões por si mesmas. Segundo o documento, é preciso que a avaliação da capacidade mental para deliberação, que pode variar ao longo do tempo, faça parte da rotina de cuidado. Até que se prove o contrário, deve-se assumir que a capacidade do paciente está preservada.

O MCA é sustentado por cinco princípios ${ }^{39}$. O primeiro deles é a presunção de capacidade: todo adulto tem o direito de tomar suas próprias decisões e deve ser considerado capaz até que se prove o contrário. $O$ segundo é o direito do indivíduo de receber apoio para tomar as próprias decisões, obtendo toda a ajuda necessária antes de que alguém conclua que ele se tornou incapaz.

O terceiro princípio é o direito de tomar decisões que podem ser vistas como excêntricas ou 
imprudentes. O quarto princípio é o do melhor interesse: tudo o que é feito para ou em nome de uma pessoa considerada incapaz deve ser de seu interesse. Por fim, o quinto princípio é o da intervenção menos restritiva: as intervenções em benefício da saúde da pessoa incapaz devem evitar ao máximo restringir direitos e liberdades básicas.

O MCA também fornece testes para avaliar a capacidade de tomada de decisão em determinada fase do desenvolvimento da doença. Esses testes seguem o critério diagnóstico ou funcional. O critério diagnóstico considera o comprometimento da mente ou do cérebro; o critério funcional tem em conta a incapacidade de entender, reter e utilizar informações ou comunicar uma decisão.

Recomendações éticas são fundamentais para que médicos e outros profissionais que lidam com pacientes com demência discutam as diretivas o quanto antes, logo após o diagnóstico ${ }^{40}$. No entanto, apesar da importância das DAD, estas ainda não são aceitas em vários estados americanos.

Sulmasy e Snyder ${ }^{38}$ aponta que as DAV construídas entre pacientes que perderam capacidade são vagas, inflexíveis, ignoradas ou não estão disponíveis. Relatam que a maioria dos pacientes não consegue antecipar os problemas futuros, e que os procuradores de saúde conseguem fazer jus aos valores do paciente em apenas dois terços das situações. Os aurores reafirmam a importância e superioridade das DAV, mas defendem um modelo substituto em que as decisões tenham o paciente como centro de cuidado em conjunto com os valores e preferências dos familiares e a decisão clínica médica. Uma possível limitação para esse modelo seria, segundo os autores, a reintrodução do paternalismo.

É necessário que profissionais de saúde e a sociedade discutam a questão da autonomia. Os profissionais de saúde, em especial, por sua proximidade com os conflitos envolvidos no cuidado de pacientes com demência, precisam se familiarizar com instrumentos que avaliam a capacidade do indivíduo para entender e avaliar as situações e expressar decisões com base em valores e preferências.

\section{Considerações finais}

A presente análise trouxe questionamentos relativos à saúde e à manutenção da autonomia na velhice, visto que, com o avançar da idade, surgem limitações e patologias como as demências, que atingem especificamente os idosos. $O$ caso mais emblemático é o da doença de Alzheimer, a mais comum entre as demências, e que devido a suas singularidades envolve muitos atores: médicos, pesquisadores, familiares, cuidadores e pacientes.

A doença de Alzheimer, que tende a ser duradoura, afeta a autonomia do idoso acometido. Por isso, recomenda-se que, ainda na fase inicial da doença, o paciente receba ajuda para planejar seu futuro. Nesse sentido, verificar a capacidade do idoso continuamente é importante para avaliar a capacidade de tomada de decisões em cada momento da enfermidade.

As DAV são uma possibilidade para que o indivíduo registre e esclareça suas opiniões, desejos e preferências em relação a procedimentos médicos e cuidados. No Brasil, porém, ainda não há legislação sobre as DAV e seus diferentes gêneros. Essa lacuna torna ainda mais necessário o cuidado de, ao confeccionar uma diretiva, não elaborar cláusulas que sejam ilícitas e, consequentemente, não possam ser realizadas.

Devido à complexidade das demências, recomenda-se que o documento de diretivas do idoso seja elaborado considerando sua doença específica. As diretivas antecipadas de vontade, então, podem ser chamadas de "diretivas antecipadas para demências". Esse documento deve ser feito assim que o idoso receber o diagnóstico, antes de que o avanço da doença comprometa sua autonomia.

Por meio das DAD, o paciente garante que suas preferências e valores relativos aos cuidados de saúde sejam respeitados na fase grave da doença, quando já não será possível se expressar. Isso diminui as preocupações com as mudanças que a doença acarreta com o passar do tempo. Cabe destacar, porém, que o documento deve ser adaptado para as várias fases das demências, considerando situações que surgem no desenvolvimento da doença e eventuais discussões entre os envolvidos no tratamento e cuidado ao paciente.

Conclui-se que é urgente debater as DAD no contexto brasileiro. Esse debate deve se dar não apenas no âmbito das instituições de saúde, mas incluir toda a sociedade, visto que se trata de um instrumento fundamental para garantir a autodeterminação de pacientes com demência. 


\section{Referências}

1. Moraes EN. Atenção à saúde do idoso: aspectos conceituais [Internet]. Brasília: Organização Pan-Americana da Saúde; 2012 [acesso 31 mar 2021]. Disponível: https://bit.ly/3ISxjqx

2. Brasil. Lei $n^{\circ} 10.741$, de $1^{\circ}$ de outubro de 2003. Dispõe sobre o Estatuto do Idoso e dá outras providências. Diário Oficial da União [Internet]. Brasília, 3 out 2003 [acesso 9 ago 2021]. Disponível: https://bit.ly/2Xdw0YQ

3. World Health Organization. Envelhecimento ativo: uma política de saúde [Internet]. Brasília: Organização Pan-Americana da Saúde; 2005 [acesso 31 mar 2021]. Disponível: https://bit.ly/3sbVOLy

4. Torres da Silva PJPC. Diretivas antecipadas de vontade: garantia constitucional da dignidade do idoso portador de Alzheimer no cenário ibero-americano [tese] [Internet]. Niterói: Universidade Federal Fluminense; 2016 [acesso 31 mar 2021]. Disponível: https://bit.ly/3s64o3m

5. Scheltens P, Blennow K, Breteler MMB, de Strooper B, Frisoni GB, Salloway S, Van der Flier WM. Alzheimer's disease. Lancet [Internet]. 2016 [acesso 9 ago 2021];388(10043):505-17. DOI: 10.1016/ S0140-6736(15)01124-1

6. Cosac DCS. Autonomia, consentimento e vulnerabilidade do participante de pesquisa clínica. Rev. bioét. (Impr.) [Internet]. 2017 [acesso 31 mar 2021];25(1):19-29. DOI: 10.1590/1983-80422017251162

7. Livingston G, Huntley J, Sommerlad A, Ames D, Ballard C, Banerjee S et al. Dementia prevention, intervention, and care: 2020 report of the Lancet Commission. Lancet [Internet]. 2020 [acesso 31 mar 2021];396(10248):413-46. DOI: 10.1016/S0140-6736(20)30367-6

8. Harrison KL, Hunt LJ, Ritchie CS, Yaffe K. Dying with dementia: underrecognized and stigmatized. J Am Geriatr Soc [Internet]. 2019 [acesso 31 mar 2021];67(8):1548-51. DOI: 10.1111/jgs.15895

9. World Health Organization. Palliative care [Internet]. 2020 [acesso 31 mar 2021]. Disponível: https://bit.ly/ 3AwsSpn

10. International Association for Hospice and Palliative Care. Palliative care definition [Internet]. 2018 [acesso 31 mar 2021]. Disponível: https://bit.ly/3AowGJ6

11. United Kingdom. National Health Service. Palliative care guidelines in dementia [Internet]. $2^{\mathrm{a}}$ ed. Manchester: NHS; 2018 [acesso 31 mar 2021]. Disponível: https://bit.ly/3lODsUu

12. Dadalto L. Testamento vital. $5^{\text {a }}$ ed. Indaiatuba: Foco; 2020.

13. Pinto ALF. Memória: um desafio à autonomia do idoso. Fam Saúde Desenv [Internet]. 1999 [acesso 31 mar 2021];1(1-2):39-48. DOI: 10.5380/fsd.v1i1.4874

14. Dell'Isola A. Mentes brilhantes: como desenvolver todo o potencial do seu cérebro. $2^{\mathrm{a}}$ ed. São Paulo: Universo dos Livros; 2012.

15. Calkins MP. From research to application: supportive and therapeutic environments for people living with dementia. Gerontologist [Internet]. 2018 [acesso 9 ago 2021];58(supl 1):114-28. DOI: 10.1093/geront/gnx146

16. Poirier J, Gauthier S. Doença de Alzheimer: o guia completo. São Paulo: MG Editores; 2006.

17. Izquierdo I, Terra N, Gomes I, Portuguez M, Myskiw J, Furini C et al. Envelhecimento, memória e doença de Alzheimer. Porto Alegre: EdiPUCRS; 2015.

18. Campos A, Oliveira D. A relação entre o princípio da autonomia e o princípio da beneficência (e não maleficência) na bioética médica. Rev Bras Estud Polít [Internet]. 2017 [acesso 31 mar 2021];(115):13-45. DOI: 10.9732/P.0034-7191.2017V115P13

19. Brasil. Lei $n^{\circ} 8.842$, de 4 de janeiro de 1994. Dispõe sobre a política nacional do idoso, cria o Conselho Nacional do Idoso e dá outras providências. Diário Oficial da União [Internet]. Brasília, p. 77, 5 jan 1994 [acesso 31 mar 2021]. Disponível: https://bit.ly/2Xe4XN1

20. Conselho Federal de Medicina. Resolução n 1.805/2006 [Internet]. Brasília: CFM; 2016 [acesso 31 mar 2021]. Disponível: https://bit.ly/3yuTqql

21. Fabbro L. Limitações jurídicas à autonomia do paciente. Bioética [Internet]. 1999 [acesso 31 mar 2021];7(1). Disponível: https://bit.ly/3yzohlv 
22. Duarte ES, Santos JJ. A equipe multiprofissional no suporte ao cuidador do portador da doença de Alzheimer. Memorialidades [Internet]. 2015 [acesso 9 ago 2021];12(23-24):89-112. Disponível: https://bit.ly/2VEAOWu

23. Brasil. Lei $n^{\circ}$ 10.406, de 10 de janeiro de 2002. Institui o Código Civil. Diário Oficial da União [Internet]. Brasília, 11 jan 2002 [acesso 31 mar 2021]. Disponível: https://bit.ly/3iuGifk

24. Brasil. Lei $n^{\circ}$ 13.146, de 6 de julho de 2015. Institui a Lei Brasileira de Inclusão da Pessoa com Deficiência (Estatuto da Pessoa com Deficiência). Diário Oficial da União [Internet]. Brasília, nº 127, 7 jul 2015 [acesso 31 mar 2021]. Disponível: https://bit.ly/3jVODKr

25. d'Alencar RS, Santos EMP, Pinto JBT. Conhecendo a doença de Alzheimer: uma contribuição para familiares e cuidadores [Internet]. Ilhéus: Editora da UESC; 2010 [acesso 31 mar 2021]. Disponível: https://bit.ly/2VITrlZ

26. Sá L, Oliveira R. Autonomia: uma abordagem interdisciplinar. Saúde Ética Justiça [Internet]. 2007 [acesso 31 mar 2021];12(1-2):5-14. Disponível: https://bit.ly/2VwlsDL

27. Karlawish JHT, Casarett DJ, James BD, Xie SX, Kim SYH. The ability of persons with Alzheimer disease $(A D)$ to make a decision about taking an AD treatment. Neurology [Internet]. 2005 [acesso 31 mar 2021];64(9):1514-9. DOI: 10.1212/01.WNL.0000160000.01742.9D

28. Schmidt AV, Tittanegro GR. A autonomia principialista comparada à autonomia do libertarismo. Rev Pistis Prax [Internet]. 2009 [acesso 31 mar 2021];1(1):173-98. Disponível: https://bit.ly/3IMdi4C

29. Andrade TFM. O papel dos cuidadores na preservação da autonomia e dignidade da pessoa com doença de Alzheimer [tese] [Internet]. Lisboa: Universidade de Lisboa; 2015 [acesso 31 mar 2021]. Disponível: https://bit.ly/2VAkplL

30. Burlá C. A aplicação das diretivas antecipadas de vontade na pessoa com demência [tese] [Internet]. Porto: Universidade do Porto; 2015 [acesso 31 mar 2021]. Disponível: https://bit.ly/3s67rbF

31. Conselho Federal de Medicina. Resolução $n^{\circ} 1.995 / 2012$. Dispõe sobre as diretivas antecipadas de vontade dos pacientes [Internet]. Brasília: CFM; 2012 [acesso 31 mar 2021]. Disponível: https://bit.ly/3xzMHdi

32. Cummings JL, Tong G, Ballard C. Treatment combinations for Alzheimer's disease: current and future pharmacotherapy options. J Alzheimers Dis [Internet]. 2019 [acesso 9 ago 2021];67(3):779-94. DOI: $10.3233 / J A D-180766$

33. Casella JT, Moreira VG. O papel das diretivas antecipadas como respaldo legal ao médico com paciente idoso com demência. Revista HUPE [Internet]. 2017 [acesso 31 mar 2021];16(2):96-101. DOI: 10.12957/ rhupe.2017.37649

34. Godinho AM. Diretivas antecipadas de vontade: testamento vital, mandato duradouro e sua admissibilidade no ordenamento jurídico brasileiro. RIDB [Internet]. 2012 [acesso 31 mar 2021];1(2):945-78. Disponível: https://bit.ly/2XeatPK

35. Assumpção VS. Diretivas antecipadas de vontade: uma análise dos limites à autonomia em função das normas penais de tutela do direito à vida [dissertação] [Internet]. Salvador: Universidade Federal da Bahia; 2014. Disponível: https://bit.ly/2VIzthn

36. Gaster B, Larson EB, Curtis JR. Advance directives for dementia: meeting a unique challenge. JAMA [Internet]. 2017 [acesso 31 mar 2021];318(22):21-75. DOI: 10.1001/jama.2017.16473

37. Alzheimer's Association. Legal plans: considerations for helping a person living with dementia plan for the future [Internet]. Chicago: Alzheimer's Association; 2019 [acesso 31 mar 2021]. Disponível: https://bit.ly/2VFX1nh

38. Sulmasy DP, Snyder L. Substituted interests and best judgments: an integrated model of surrogate decision making. JAMA [Internet]. 2010 [acesso 31 mar 2021];304(17):1946-7. DOI: 10.1001/jama.2010.1595

39. United Kingdom. Mental Capacity Act 2005. Legislation.gov.uk [Internet];Section 16A. 2005 [acesso 20 jul 2021]. Disponível: https://bit.ly/3xUrTgZ

40. Burlá C, Rego G, Nunes R. Alzheimer, dementia and the living will: a proposal. Med Health Care Philos [Internet]. 2014 [acesso 31 mar 2021];17(3):389-95. DOI: 10.1007/s11019-014-9559-8 
Luciana Dadalto - Doutora - luciana@lucianadadalto.com.br (D) 0000-0001-5449-6855

Alexandra Mendes Barreto Arantes - Especialista - alexandragericp@gmail.com (iD) 0000-0001-7195-9356

Priscila Demari Baruffi - Especialista - priscila_baruffi@yahoo.com.br (iD) 0000-0001-6329-3136

\section{Correspondência}

Priscila Demari Baruffi - Rua Borges de Medeiros, 37, apt. 202, Centro CEP 95720-000. Garibaldi/ RS, Brasil. 